hundred per cent no-touch technique of both surgical and theatre staff is essential in all bone operations, and especially in those which include grafting. Deaths are still occurring secondary to bone grafting operations and are due to sepsis.

Another feature, which this monograph demonstrates, is the difference in the type of fracture occurring in the Royal Air Force and in those occurring in civilian life. Mr. Armstrong found that it was necessary to graft $6.54 \%$ of a total of $1,16 r$ fractures. This is from fifteen to twenty times the number which require grafting in a civilian fracture clinic.

Mr. Armstrong has developed his technique of grafting fractures of the carpal scaphoid almost to perfection. Readers are well advised to recognise that this is a very difficult operation to perform. Many orthopaedic surgeons, however, will question his procedure of grafting all "un-united" fractures of the scaphoid after only three months immobilisation. After three months of such treatment, fractures of the scaphoid are frequently united although X-ray pictures suggest a gap. A similar X-ray gap with the appearance of non-union occurs frequently in clinically united fractures of the tibia.

This monograph advises the use of the onlay graft for delayed union in fractures of the shaft of the tibia. Many orthopaedic surgeons still find, for various reasons, that an inlay graft is preferable.

There is little doubt that in civilian life bone grafting is seldom required, provided the primary reduction and immobilisation are efficient. There are two noteworthy exceptions, namely, fractures of the lower one-quarter of the shaft of the humerus, and fractures of the lower shaft of the radius with dislocation of the lower extremity of the ulna. Furthermore, the monograph. has not stressed the fact that in war wounds, associated with compound fractures of the shafts of the radius and ulna, bone grafting is greatly assisted by preliminary skin grafting with a full thickness graft.

Mr. Armstrong is to be congratulated on demonstrating the excellent results of one school of orthopaedic procedure in the treatment of fractures. He has also re-affirmed the need for a perfect mechanical and no touch aseptic technique in order to render the operations of bone grafting successful.

\section{PHYSICAL MEDICINE IN GENERAL PRACTICE}

By Dr. William Bierman, M.D., Hamish Hamilton Med. Books. Price 37s.

It is a little difficult for me to entirely comprehend for which type of practitioner this book is written, although it is dedicated to the practitioner in general practice in the United States of America, certainly no practitioner in this country could deal with the many technical problems raised in this book, whereas from a physical medicine specialist's point of view the book is very unbalanced. There is very little detail about electrical apparatus. There is too much on experimental pathology, yet not enough on the actual mechanical details of giving physical treatment. The chapter on massage is extremely sketchily done. The illustrations throughout the book are extremely mediocre and disappointing and there is a marked absence of pictures illustrating electrical apparatus. The chapters on the Application on Physical Medicine to the Various Systems and Diseases is very helpful, but is hardly full enough.

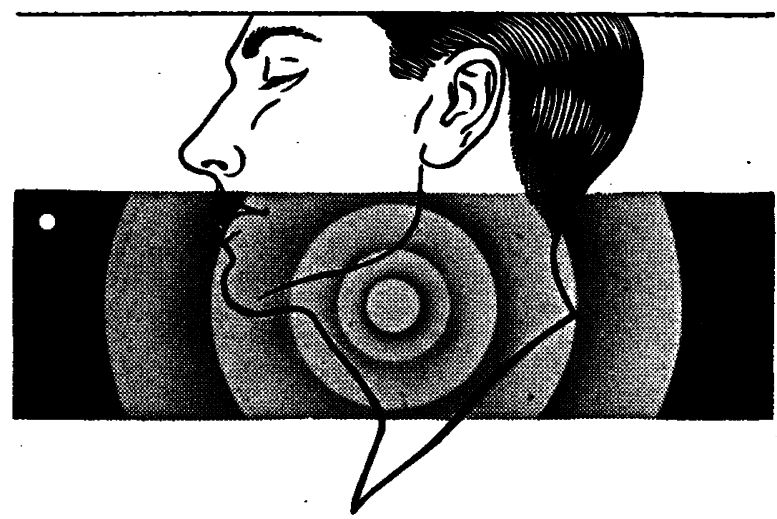

A course of general irradiation with ultra-violet rays often provides 'the initiative or dynamic key which sets in motion the physiological process of recovery' (writes a County M.O.H.). Physicians of all lands accept the Hanovia Alpine Sun as the standard ultra-violet ray lamp in hospital or consulting room use. The current Model VII Alpine Sun is shown above in use at a Government Rehabilitation Centre.

\section{SOME CLINICAL USES OF ALPINE SUN LAMP:-} THE

Surgery: Wounds that are slow in healing respond well to local irradiation. The re-calcifying effect of general exposure is beneficial in fractures.

Skin Diseases: Ultra-violet irradiation is markedly beneficial in acne, impetigo, psoriasis, and many other conditions. It greatly assists in restoring a healthy condition of the skin following eczema or dermatitis.

Burns, scalds, abrasions: Painless healing and good cosmetic results follow local and general irradiation.

Health: "Ultra-violet radiation is a most valuable measure for safeguarding the health of workers. It is thus a boon the use of which should be most widely encouraged" (writes an Industrial Medical Officer).

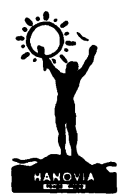

HANOVIA LTD

Specialists in actinotherapy equipment SLOUGH

Showrooms:

3 Victoria Street, London, S.W.1
Prospectus No. 135 giving details of the Alpine Sun and other equipment for actinotherapy is (please send 1d. stamp four copy).
your
Mr 35B/20 